\title{
Scanning Electron Microscopy of Lung Capillary Casts of Caveolin-1 Knockout Mice Show Extensive Architectural Distortion
}

\author{
N.A. Maniatis, * D.E. Schraufnagel*, A.B. Malik**, R.D. Minshall**§ \\ * Department of Pulmonary, Critical Care and Sleep Medicine, University of Illinois at \\ Chicago, 840 S. Wood St., Chicago, IL 60612 \\ **Department of Pharmacology, College of Medicine, University of Illinois at Chicago, \\ 808 S. Wood St. Chicago, IL 60612
}

$\S$ Department of Anesthesiology, University of Illinois at Chicago, $808 \mathrm{~S}$. Wood St. Chicago, IL 60612

Background and Aims: Caveolae are 50-100 nm vesicles, which originate from cholesterol- and sphingolipid-rich cell membrane domains called lipid rafts. In addition to harboring many cell signaling molecules on their surface, they also transport albumin and other molecules across the endothelium, a function termed transcytosis. Caveolin-1, the defining protein of caveolae, is abundantly expressed in these structures. It forms a scaffold on the surface of caveolae and maintains their structure and function through interactions with multiple other proteins.

Caveolin-1-/- mice $\left(\mathrm{CAV1}^{-/}\right)$lack caveolae and show extensive histologic abnormalities in the lung including alveolar septal thickening, hypercellularity and fibrosis. They have been shown to have markedly increased levels of nitric oxide, yet they show elevated pulmonary arterial pressures and right ventricular hypertrophy. In the present study we investigate the effects of caveolin-1 deletion on the pulmonary arterial microvasculature using vascular corrosion casting.

Methods: 4 male Swiss Black caveolin-/- mice aged 3 months and aged-matched controls were anesthetized with intraperitoneal injection of Ketamine $120 \mathrm{mg} / \mathrm{kg}$-Xylazine 16 $\mathrm{mg} / \mathrm{kg}$. Heparin 500 i.u. was administered via the same route. Lethal hemorrhage was induced by inf. V. Cava disruption. The body of the mice was then transected at the level of the lumbar spine, a non-beveled 20 gauge needle was inserted in the thoracic aorta and secured with a suture. Partially polymerized methyl-methacrylate (Mercox, Ladd Research Imdustries, Burlington, VT. USA) was injected in the aorta over 1 minute until retrograde filling of the lungs was evident or $3 \mathrm{ml}$ were injected. One hour was allowed for polymerization of the sclerozing agent. The lungs were removed and placed in a $\mathrm{NaOH}$ solution at $37 \mathrm{C}$ until all the tissue was completely digested. One mm thick sections of the lungs were mounted on aluminum studs using double sided tape, sputtercoated with palladium-gold and viewed with a JEOL 35C scanning electron microscope.

Results: Pleural and cut surfaces of the wild type control and knockout animals showed complete filling of the vasculature into the level of the capillaries. While in the wild type mice the vascular casts appeared very smooth, cylindrical and of even size, in the knockout animals there was extensive architectural distortion consisting of an irregular, 
"ruffled" appearance of the capillaries as well as several areas of flat appearing casts consistent with capillary collapse (figure). The ruffles were about $1 \mu \mathrm{m}$ in diameter and ran with the length of the capillary. Whether these are compressions from inter-alveolar collagen, which is increased, or a form of beginning angiogenesis remains to be determined.

Conclusion: Genetic deletion of the caveolin-1 gene causes extensive architectural distortion of the pulmonary microvasculature. Vascular corrosion casting of these deformities may explain the pulmonary hypertension observed in these animals.

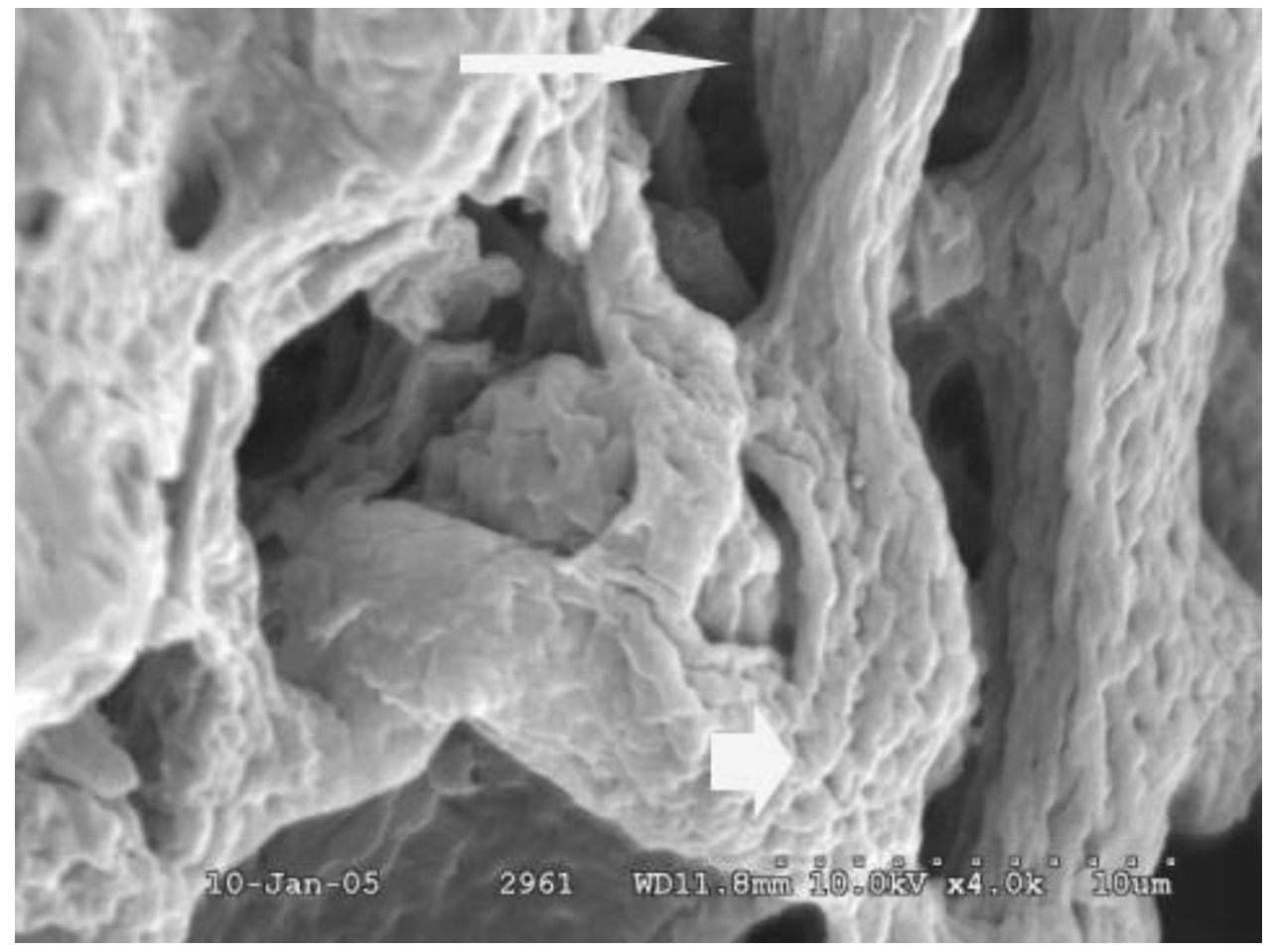

Figure: Caveolin-1 knockout mouse: Scanning electron microscopy of a corrosion cast preparation of pulmonary capillaries shows marked irregularity (thick arrow) and flattening/torsion (thin arrow) of the capillary lumen.

\section{Reference:}

[1] Zhao YY et al., Proc Natl Acad Sci U S A. 2002 Aug 20;99(17):11375-80. 